\title{
GRAPHICAL USER INTERFACE PERSONALIZATION: USER STUDY OF IMAGE FREQUENCY PREFERENCES
}

\author{
Malwina Dziśko ${ }^{1}$, Anna Lewandowska ${ }^{1}$ and Anna Samborska - Owczarek ${ }^{2}$ \\ ${ }^{1}$ West Pomeranian University of Technology, Żotnierska 52, 71-210 Szczecin, Poland \\ ${ }^{2}$ FRIS - Thinking Styles, Wojska Polskiego 150/1, 71-324 Szczecin, Poland
}

\begin{abstract}
Recommending systems are undoubtedly a breakthrough in technology. Recommending systems are helpful to produce intelligent systems. These systems are becoming more and more popular and their main components are other embedded subsystems. The final result of intelligent system visible to the end-user is the graphical interface, which mainly consists of the graphical representation. It turns out, that wrongly chosen image frequency, to detail, chaotic and so, can affects the user's work efficiency. Thus, the image frequency is one of the components that should be investigated during graphical interfaces design. The main purpose of this work is to analyze the impact of the selection of the frequency level of images depending on the human thinking styles. The paper presents the selection of images with given frequency depending of one thinking style. To test the research hypothesis, we conducted perceptual experiments in which images with different intensity in different frequency bands were presented. Finally, we obtained statistical significance between the selection of images with different levels of frequency bands of images for the group of respondents with the thinking style based on the Ideas and the rest of the thinking groups.
\end{abstract}

\section{KEYWORDS}

Intelligent Graphical User Interface, Frequency Bands Images, Human Thinking Style, Perceptual Experiment - User Study

\section{INTRODUCTION}

Recommending systems become more and more popular. These systems make tight integrations of the calculation layer and physical processes. They are used more and more almost everywhere. They most often occur in the form of embedded systems (Lakshmi et al., 2019; Masayoshi et al., 1993). It controls physical processes that are further data sources for calculating the object control signal., They are considered as breakthrough not only in IT but also in other industries (Keenle et al., 2000). Thanks to these systems, it is possible to use the Internet of Things technology, where it is necessary to use even signals from various sensors and measuring devices (Field, 1989).

Intelligent systems are also important from the point of view of computer graphics interfaces and what follows of the final version of a graphic interfaces for the user - intelligent graphic interfaces preparation (Michael, 2000). Since physical Cyber systems occur in the form of a built-in embedded system in the final system, the graphic interface is finally visible as the output for the potential user (Kozhevnikov, 2007). It should be mentioned here, that one of the potential problem is preparing such an interface properly designed for a specific group of users, e.g. doctors, pilots, etc. Thus, incorrectly designed such an interface may affect the user's work efficiency with interface. It also turned out that the choice of user preferences is also influenced by the individual pattern of the user's perceptual, often called the thinking style (Field 1989; Zetzsche et al., 1989). Thus, it can be concluded that the design of graphic interfaces should take thinking style into account.

We distinguish many components by means of which we create an user interface. Among others the color of the objects displayed on the screen, the way objects are arranged on the screen, but also the complexity of the graphics used (Rossi et al., 2019).

As already stated, the contrast of image affects the user's choice of image. Thereby frequency of the images used in graphical interface could affect user's efficiency with this interface. In our paper, we decided to present the relationship between individual selection of images with different image frequency 
(„Frequency determines the number of cycles of the periodic phenomenon occurring per unit of time. The frequency of 1 hertz corresponds to the occurrence of one cycle in 1 second. The $\mathrm{Hz}$ unit is not used when describing the image frequency. The image frequency is described in cycles per unit of distance" (Suchwałko et al., 2007)) - actually, the frequency bands of frequency and their thinking style. Finally, we show how this translates into creating graphic interfaces. We used perceptual psychometric model created by FRIS® in order to check thinking style of experiment respondent (Lewandowska et al., 2018; Dziśko et al., 2018).

To test the hypothesis - that the image frequency affects the selection of images by users, we conducted perceptual experiments using images with different content. The main goal of our work, is to analyze the impact of the level of image frequency and thinking style of a human observer. The problem was mentioned in the literature. However, in the literature, only the level of global contrasts of images is presented which does not present dependence of these contrasts on the thinking style of a human being. Therefore, this paper takes into account not only the global but also the local contrast of the displayed images and how it relates with the human thinking style (Lewandowska et al., 2018; Dziśko et al., 2018).

The obtained results were submitted to statistical analysis, which showed that there is a strong correlation between the selection of the image with a certain frequency and the human thinking style. Thus, the results confirmed that the human thinking style is strongly correlated with the individual choice of images with different frequency bands.

Conducted research are important from the computer graphics point of view, because they can further improve designing intelligent user interfaces, by taking on board one natural preferences. Since people differ one from another, their work efficiency with graphic interface may be varied. Some people reacts negatively when looking on images containing many intricate elements. For that reason, many graphical details in interfaces dedicated to totality of users should be avoided.

The research was carried out on images depicting natural, real life scenes to provide all users with the same level of knowledge in research. The experiments were deliberately not performed on the interfaces because some people could be familiar with them, thus they would not respond in a natural way, but learned.

Thanks to such kind of approach, it is possible to create a system that automatically selects interface complexity of graphics for user interface in relation to thinking style. This allows various social groups to use the intelligent interface, thereby improve their work with the system.

The concept of an experiment is described in Section 3. Section 5 explains in detail the subjective experimental procedure that we used. The results and analysis can be found in Section 6. Finally, Section 7 concludes the paper.

\section{RELATED WORK}

The popularity of recommending systems is recently growing up (Zetzsche et al., 1989). They are used in various industries ranging from used, ranging from building intelligent houses through in medicine (Stoppe et al., 2019; Zhang 2019). They are designed to facilitate the work of the human. The final kind of such system consist of large amount of embedded smaller sub-systems (Zetzsche et al., 1989; Zhang 2019). Final output visible to the end-user is a graphical interface which consists of among others displayed images.

It turns out, that an appropriate selection of frequency of images in graphic interface can improve the work of the human, while using interface(Zetzsche et al., 1989). The basis for such selection is not only the content of images, but also the level of their frequency. Literature indicates that the image frequency is the medium of visual information (Zetzsche et al., 1989; Rossi et al., 2019).

It is considered, that images with higher contrasts are more appealing to users, but they are less natural., So the higher the contrast, the more unnatural the picture. However, it should be taken into account that every person is an individuality, who can perceive and search the area of images in various ways (Zetzsche et al., 1989).

Graphical user interface. There are a lot of various user's interfaces that people are using day by day. As end-users we expect from them high effectiveness and intuitivness. However, it would be valuable when design of those interfaces would take into account scientific knowledge about human thinking styles. Stuart Card in his book (Card, 2017), argue that analysis of information processing will lead to new methods, which will be using thinking psychology in order to creating interfaces. He suggests that abovemnetioned analysis will lead to new ways to adapt psychology to our human needs. He also points out that in the studies of human-machine systems and engineering psychology for some time it has been common to include a flowchart of the entire human information processing system (Card, 2017). In the other hand, Aude Dufresne in (Dufresne et al., 1997) discusses the problem of the impact of human thinking styles on navigational 
strategies referring specifically to hypermedia. The author of this text draws attention to the knowledge of thinking styles of a human being, referring to the style of learning of users (Dufresne et al., 1997). However, in these papers, none of the authors specifies any human thinking styles.

Therefore, in this work, we decided to use an approach in which we used the human's thinking style for analysis. Thanks to this, it is possible to check the correlation between choosing a picture with the right level of frequency and the thinking style of a human being. Such kind of dependence will translate into creation of recommending graphical interfaces that could support automatic selection of graphic contrast usage in the user interface which is relative to the thinking styles.

Currently, it is difficult to find research that would check the impact of human thinking style on the creation of recommended user interfaces, while taking into account the selection of appropriate image frequency bands.

Therefore, we decided to carry out research taking into account human thinking style, including dependence on the field and independence from the field.

By dependence on the field, we understand the tendency to perceive holistic, in which the elements constitute the picture of the whole. By independence from the field, we mean the tendency to separate the components and make them and make them into parts - as if they are independent from the whole. Independence from the field means perceiving analytically, dependence from the field is perceiving globally (Dufresne et al., 1997).

\section{CONCEPTION}

The aim of the paper was to examine whether the human thinking style influences the human attention on the graphical components depending on their complexity (details level - high contrast in a particular frequency band). Therefore our research was divided into two parts. The first is a perceptual experiment that was conducted. In the second part the observers were asked to complete the FRIS ${ }^{\circledR}$ psychometric questionnaire. After that we obtained the complete data we carried out the analysis. The diagram of the work is presented in the figure below (see Figure 1.).

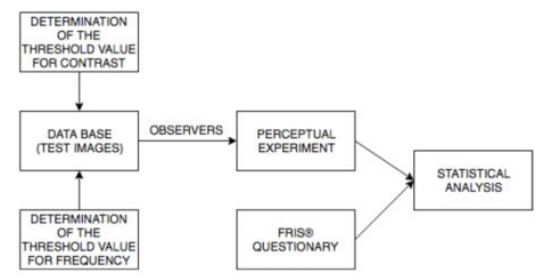

Figure 1. Preparation of the perceptual experiment and the process of gathering knowledge

\section{HUMAN THINKING STYLES}

The style of thinking is innate, and man acquires it from birth. Due to the fact that the style of thinking is innate, the preferences that a person acquires along with his style of thinking since birth are subconscious. Of course, people acquire subjective preferences with age, but these preferences correlate with the style of thinking that was already acquired at birth.

According to the literature, there are many approaches that aim to describe and determine the human thinking style (Kozhevnikov, 2007). Most of these approaches indicate bipolar scales. These are mostly: the reflection coefficient and impulsivity (Kozhevnikov, 2007). There are also thinking styles that are individual differences of every human being. These styles are measured using formal questionnaires or inventories. In our experiment, we used the FRIS $®$ inventory to verify the thinking styles of the respondents. FRIS $®$ is a psychometric questionnaire measuring the ranking of individual preferences. FRIS ${ }^{\circledR}$ defines 4 thinking perspectives:

- F: fact-based thinking (concrete, sequential, logical thinking)

- R: relation-based thinking (intuitive, field-dependent, relational thinking)

- I: Idea-based thinking (abstract, lateral, global thinking)

- S: structure-based thinking (field-independent, reflective, analytical thinking) (Samborska-Owczarek 2019A). 
FRIS $®$ is psychometrically reliable, valid, normalised and standardised. The 6 factors of the inventory are bipolar scales discriminating between pairs of thinking perspectives: $F$ vs I, F vs $\mathrm{R}$ etc. with internal consistency $0.76-0.82$ Cronbach's $\alpha$. The 4 superfactors 'internal consistency is $0,82(\mathrm{~F}), 0,88(\mathrm{R}) 0,88(\mathrm{I})$ $0,88(\mathrm{~S})$ Cronbach's $\alpha$. As a metrics in our experiment we used 1 nominal variable T S Thinking Style - the dominant (max-imal) thinking perspective of the subject (one from the set): $T S=\{F, R, I, S\}$ (Samborska-Owczarek 2019; Samborska-Owczarek 2019A).

\section{SUBJECTIVE EXPERIMENT}

During the experiments, we checked whether the contrast level in a given frequency band and the human thinking style influences the selection of images by the user. Observers were asked to read the written instructions before the experiment.

Experimental procedure. We collected data through method called forced selection (Mantiuk et al., 2012). Observers were always forced to choose one of the pictures, even if they did not see the difference between them (Mantiuk et al., 2012). There was no time limit to do the experiment. In order to avoid observer fatigue the session was prepared in such a way that it took no longer than 30 minutes. The experiment was conducted using an eye-tracker that allows later to analyze the way the observers perceive displayed images.

Observers. In the experiment, we examined 201 respondents with normal or corrected for normal vision. The age of observers varied between 20 and 70. We ask observers to select the image visible in the image according to the given command.

Display conditions. Experiment was performed using the NEC monitor with a native resolution of 1680x1050 pixels. The display conditions were measured with a Minolta CS-200 colorimeter and Specbos 1201 spectroradiometer. The measurements were used to calibrate display and ensure that all images were reproduced in the sRGB color space. We also used an eye-tracker in order to follow the path of the respondents' eyes during experiment session.

Test images. The experiment was conducted on 15 images taken from Fotolia database (subset of an image set composed for FRIS® project (Samborska-Owczarek 2019A).) ( see Figure. 2.) with different contrast levels (low, medium, high). To perform thresholding we used following procedure: first we calculate the contrasts for all images, then create a scale interval for each contrast range, and finally calculate the levels of the next 6 octaves of the global contrast. During single experiment session images were displayed in random order. To each of the images we attached one research task: "indicate the image, the most similar to the middle one" or "indicate the image that you like the most". Between each images presentation we displayed grey background without any images on it.

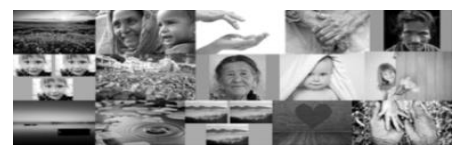

Figure 2. Test images taken to the experiments

Thereshold of frequency and contrast values. Frequency and contrast values were set to three thereshold levels: low, medium and high. In order to do this this we calculated the values of contrasts and values for individual frequency bands. Then we find the highest and the lowest value of frequency band of contrast. We separated domain ranking from highest and lowest contrast frequency into 3 parts. We assigned following labels for each part: Low, Medium, High. Finally, we match each contrast value from out data into one of three previously mentioned groups (see Figure. 3.).

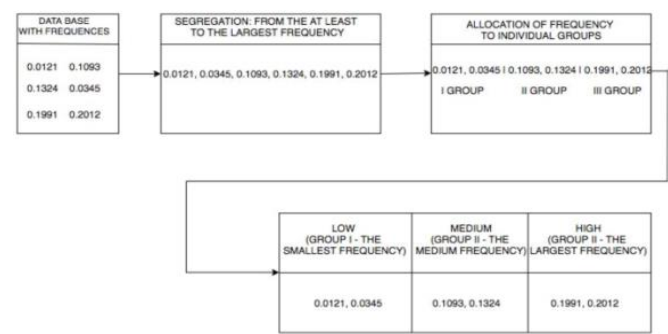

Figure 3. Example frequency distribution 


\section{RESULT AND ANALYSIS}

After the experiment we carried out a statistical analysis. Statistical analyzes have shown that there is a statistical significance between the style of human thinking and the frequency of the image chosen by the respondent.

The obtained results suggest that differences in preferences between groups of respondents of various thinking styles are noticeable. Especially between the choice of respondents whose thinking style is based on Ideas and other groups of thinking styles (Fact, Relations and Structure).

Thanks to a large research group, we found that the difference between groups is statistically significant. The experiment was completed by 201 respondents. Numerosity of people based on different thinking thinking styles were following: Ideas (I) - 33, Facts (F) - 28, Relationships (R) - 40, Structures (S) - 100.

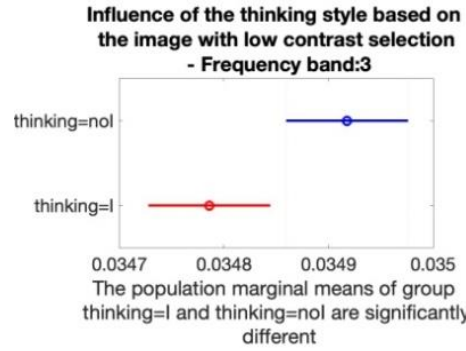

Figure 4. ANOVA comparison of observers preferences given for low frequency, for thinking style Idea-based thinking group and the rest of thinking groups. - Third band, where: I - Thinking style based on Idea, noI - other thinking styles than Idea

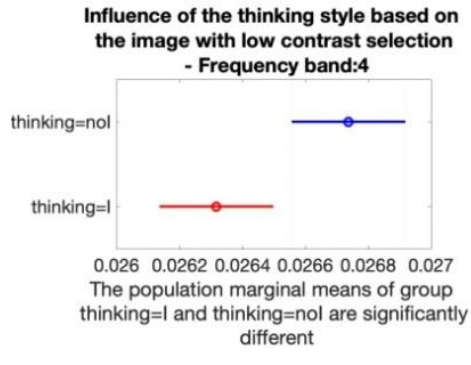

Figure 5. ANOVA comparison of observers preferences given for low frequency, for thinking style Idea-based thinking group and the rest of thinking groups. - Fourth band, where: I - Thinking style based on Idea, noI - other thinking styles than Idea

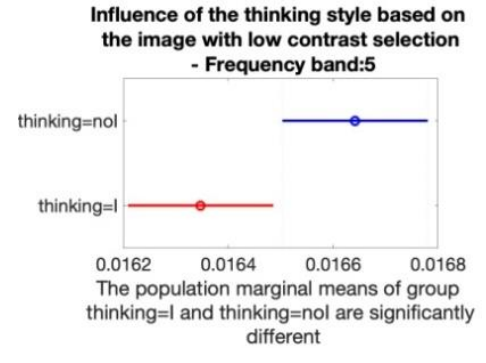

Figure 6. ANOVA comparison of observers prefeences given for low frequency, for thinking style Idea-based thinking group and the rest of thinking groups. - Fifth band, where: I - Thinking style based on Idea, noI - other thinking styles than Idea

Plots presenting achieve statistical significance are shown on Figure 4-6. Performed ANOVA analysis showed that, we obtained statistical significance between the groups of respondents based on the thinking style of the Ideas and the selection of images with low contrast. Particularly high correlation can be seen in low contrast images and low frequency bands(octaves): third, fourth and fifth. Statistical significance is changed as follows: for 3rd frequency band: $p=0.0241$, 4th frequency band: $p=0.0241$, 5th frequency band: $\mathrm{p}=0.0388$. Below in Table 1 also shows the results for thinking styles other than Ideas based. The table presents results only for low contrast, because for high and medium levels of contrast, we did not notice statistically significant data.

Table 1. Statistical significance for all cognitive styles - Low contrast

\begin{tabular}{lccc}
\hline $\begin{array}{l}\text { Thinking } \\
\text { Styles }\end{array}$ & $\begin{array}{c}\text { Level of statistical } \\
\text { significance for low contrast } \\
\text { for frequency band } \mathbf{3}\end{array}$ & $\begin{array}{c}\text { Level of statistical significance for low } \\
\text { contrast for frequency band } \mathbf{4}\end{array}$ & $\begin{array}{c}\text { Level of statistical } \\
\text { significance for low } \\
\text { contrast for frequency } \\
\text { band 5 }\end{array}$ \\
\hline Ideas & 0.0241 & 0.0241 & 0.0388 \\
Facts & 0.1231 & 0.1427 & 0.1244 \\
Structures & 0.2332 & 0.1622 & 0.2103 \\
Relations & 0.0621 & 0.0556 & 0.0677
\end{tabular}




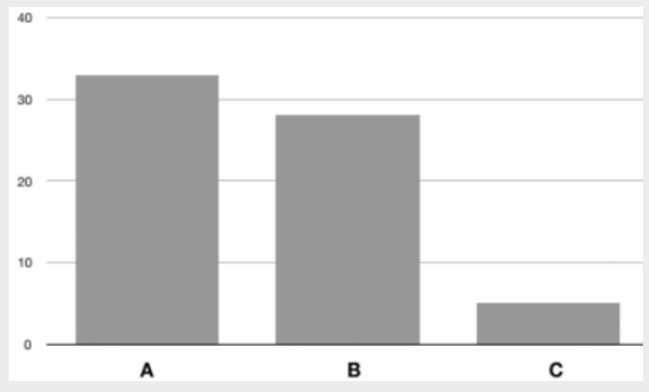

Figure 7. Quantitative analysis - groups based on thinking styles is Idea and other group. A - All respondents based on all group. B - All respondents based on Idea

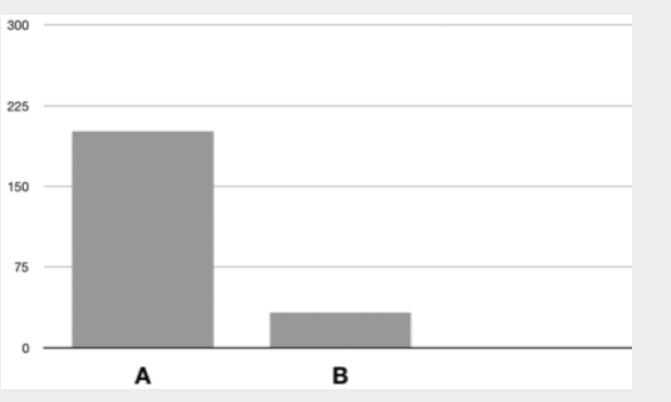

Figure 8. Quantitative analysis - groups based on Ideas. A - All respondents based on thinking styles is Idea.

B - Respondents based on Ideas who chose low frequency bands. C - Respondents based on Ideas who chose other than low frequency bands

Subsequently, we conducted a quantitative analysis see Figure 7-8. It turned out that the greatest relationship is visible between groups based on Ideas and other groups. 33 respondents on the background of the whole group (see Figure. 7 A) are people based on a group of Ideas (see Figure. 7 B). It turns out that 28 respondents (see Figure. $8 \mathrm{~B}$ ) from the group of 33 respondents based on a group of thinking styles is Ideas (see Figure. $8 \mathrm{~A}$ ) chose images with low frequency bands see Figure 8 . This is up to $85 \%$ of compatible choices. Thanks to data gathered from the eye-tracker, it turned out that users with an Idea- based thinking style focus on objects that are less detailed and less blackened. It is a visually confirmed by the ANOVA statistical analysis that respondents whose thinking style is based on relationships prefer low-frequency images (see Figure 9-10.).
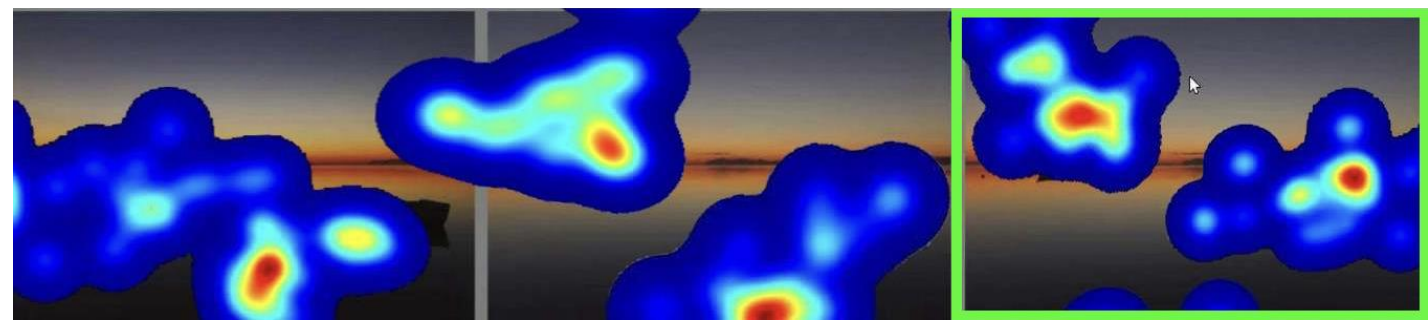

Figure 9. Heat map. Groups based on Ideas. Image with low frequencies. Green frame - the image chosen by the observer
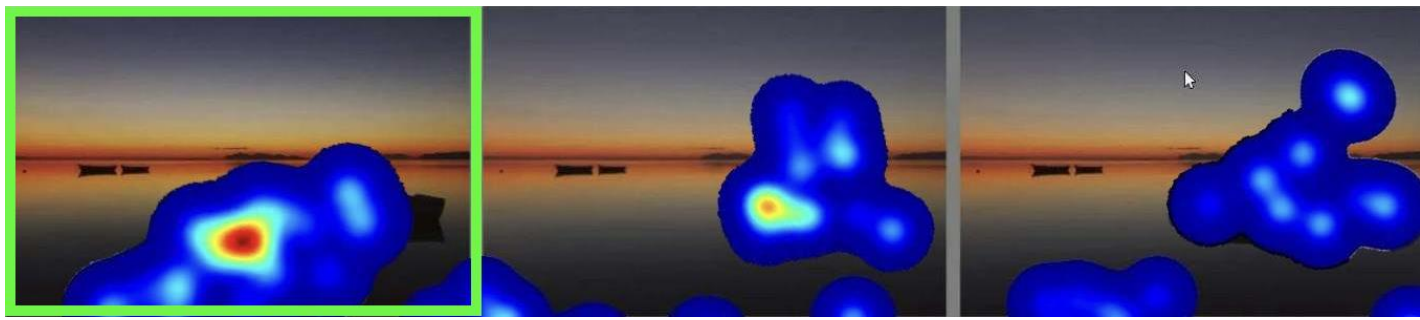

Figure 10. Heat map. Groups based on other thinking styles (Thinking styles: Relation, Fact and Structure). Image with low frequencies. Green frame - the image chosen by the observer (Observers which thinking styles was: Relation, Fact and Structure) 

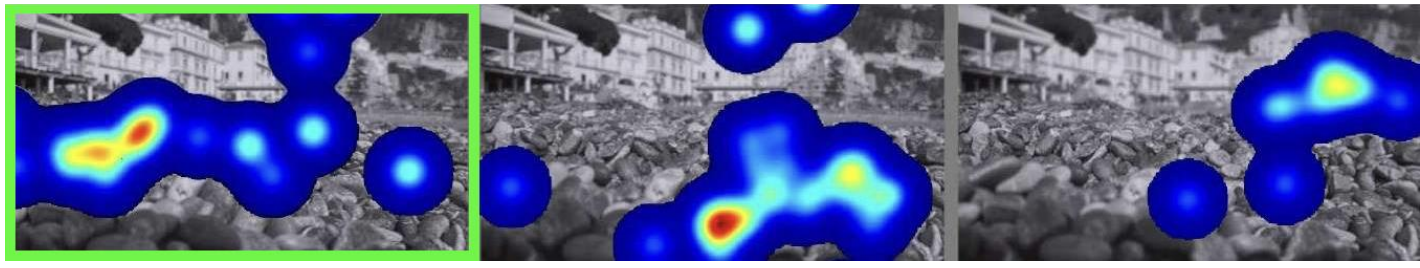

Figure 11. Heat map. Groups based on Idea thinking styles. Image with high frequencies. Green frame - the image chosen by the observer. (Observers which thinking styles was: Idea)
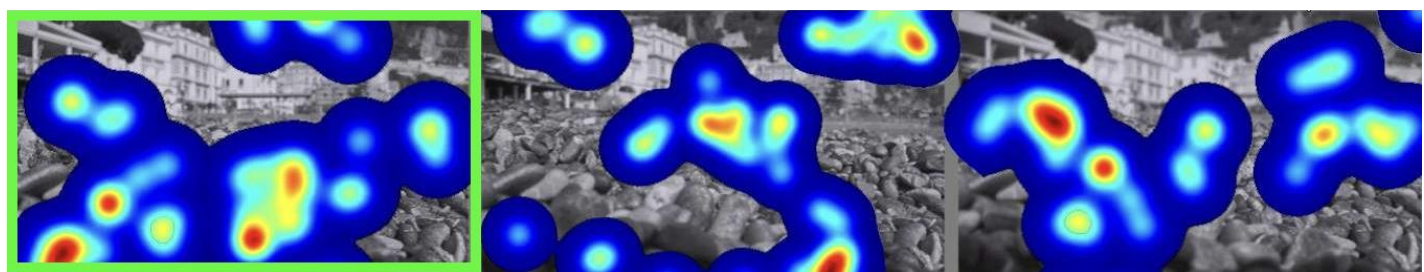

Figure 12. Heat map. Groups based on other thinking styles (Thinking styles: Relation, Fact and Structure). Image with high frequencies. Green frame - the image chosen by the observer (Observers which thinking styles was: Relation, Fact and Structure)

Visual analysis, thanks to using the eye-tracker, also showed that other thinking styles (based on relationships, structures and facts) focused on high or medium-frequency images (see Figure 10-12.). However, in this case we did not obtain statistically significant results.

Through the visual analysis, we also observed the time of focusing on images by observers. It turned out that Idea-based groups focus their attention on low- frequency images for an average of 9.2 seconds, while on high-frequency images the average focus time of these groups is 6.5 seconds. It is also noticable, that groups based on other styles than Ideas have focused their attention on high-frequency images longer - the average time is 8.4 seconds, with average focal length on low- frequency images of 6.1 seconds. It arise from the dependence on the fields based on Ideas (Lewandowska et al., 2018).

The most important result of this study, is the fact that there is a difference between the selection of low-contrast images and frequency bands for the group of respondents with the thinking style based on the Ideas and the rest of the thinking groups. That means, that respondents whose thinking style was based on Ideas choose much more often a picture with a low contrast and low frequency bands level than the rest of the respondents whose thinking style was based on the other FRIS® thinking styles. This indicates other groups prefer images with a low contrast level with a low frequency bands.

\section{CONCLUSIONS}

The main purpose of this work is to check whether human cognitive style affects human preferences related to the frequency of images. It turned out, that people with a thinking style based on Ideas choose images with low frequency in the third, fourth and fifth contrast bands.

Analysis show, that we should choose the images with high contrast in low frequency bands. Otherwise, it may turn out that the user who will work with the interface unsuitably prepared for his natural preferences will be less efficient and his frequency of errors will increase.

It is worth noting that many people are unaware of their thinking style, but the important thing is that thinking style is innate. Due to the fact that the style of thinking is innate, a person with a specific style of thinking acts subconsciously and in accordance with this style of thinking. Therefore, creating interfaces that will harmonize with the style of thinking is important because it will make the subconscious work easier with these interfaces. 
Performed analysis confirmed the hypothesis. We have obtained statistically significant results, in particular between choices of a low contrast for its various octaves and a group of people, who according to the psychometric model of FRIS $®$, have the Ideas activated.

We obtained statistically significant results for a group of respondents with a thinking style based on Ideas.

The research included in this paper may refers into the creation of personal intelligent graphical interfaces that will automatically select the level of contrasts of images and frequency bands for people with a specific thinking style. By using the recommending system, which will automatically adjust the level of contrast to human thinking thinking style, it is possible to increase the efficiency of one work.

\section{REFERENCES}

Card, S., (2017), The psychology of human-computer interaction.

Dufresne, A., Turcotte, S., (1997), Thinking style and its implications for navigation strategies. Artificial intelligence in education knowledge and media learning system, 287-293.

Dziśko, M., Lewandowska, A., \& Samborska-Owczarek, A., (2018), Does the Research Question Structure Impact the Attention Model? User Study Experiment. In International Conference on Computer Vision and Graphics, pp. 487-498.

Field, D., (1989), What the statistics of natural images tell us about visual coding. In: Proceedings of the SPIE, pp. 269-276.

Keeble, R., Macredie, R., (2000), Assistant agents for the world wide web intelligent interface design challenges. Interacting with computers, pp. 357-381.

Kozhevnikov, M., (2007), Thinking styles in the context of modern psychology: toward an integrated framework of thinking style, pp. 464- 481 .

Lewandowska, A., Samborska-Owczarek, A., \& Dziśko, M., (2018), Contrast Perception Across Human Thinking Style. In International Conference Image Analysis and Recognition, pp. 345-352

M. Lakshmi, et al., (2019), Navigation Through Eye-Tracking for Human-Computer Interface. In: Information and Communication Technology for Intelligent Systems, p. 575-586.

Mantiuk, R., Tomaszewska, A., Mantiuk, R., (2012), Comparison of four subjective methods for image quality assessment. In Computer graphics forum, pp. 2478-2491.

Masayoshi, M., et al., (1993), Interactive intelligent interface., pp. 5,201,034.

Michael F., (2000), Intelligent interface technology: from theory to reality?. Interacting with computers, pp. 323-336.

Rossi M., Sani S., Nibali M. Fornia L., Bello L., Byrne R., (2019), Mapping in Low-Grade Glioma Surgery. Low-and High-Frequency Stimulation, pp. 55-63.

Samborska-Owczarek, A., (2019), FRIS Thinking Styles Inventory, https://fris.pl

Samborska-Owczarek, A., (2019), FRIS Thinking Styles Inventory., https://fris.pl/ FRIS-validity-data.pdf

Stoppe, L., Husemann, C., (2019), U.S. Patent Application, pp. 175-468.

Suchwałko A., Wysocka-Król K., (2007), Politechnika $\quad$ Wrocławska, http://www.dydaktyka.ib.pwr.wroc.pl/materialy/ETP002901\%20Diagnostyka\%20obrazowa/Ćwiczenie_6.pdf

Zetzsche, C., Hauske, G., (1989), Multiple channel model for the prediction of subjective image quality. In: Proceedings of the SPIE, pp. 209-216.

ZHANG P., (2019), Objective method for assessing high contrast resolution of image based on Rayleigh criterion and testing operating method. U.S. Patent Application No 10/169,870. 